


\section{MEDIENEPISTEMOLOGIE und KINOARCHÄOLOGIE in aktuellen Publikationen}

Besprochen von PETRA LÖFFLER

François Albera, Maria Tortajada (Hg.), Cinema Beyond Film. Media Epistemology in the Modern Era, Amsterdam (Amsterdam Univ. Press) 20 Io.

Pasi Väliaho, Mapping the Moving Image. Gesture, Thought and Cinema I9oo, Amsterdam (Amsterdam Univ. Press) 2010.

Die von Thomas Elsaesser seit 1994 im Amsterdamer Universitätsverlag herausgegebene Buchreihe Film Culture in Transition umfasst mittlerweile 29 Publikationen, darunter zahlreiche Studien zum amerikanischen und europäischen Kino, zu einzelnen Regisseuren, lokalen Filmkulturen und Genres sowie zu übergreifenden medientheoretischen Fragen. Auch Siegfried Zielinskis Mediengeschichte der Audiovisionen ist hier in englischer Übersetzung erschienen. Elsaesser selbst ist als Autor und Herausgeber prominent vertreten. Die vorerst letzten beiden Bände der Reihe widmen sich dezidiert der Archäologie und Epistemologie des Films/Kinos.

Medienarchäologische Untersuchungen haben seit geraumer Zeit in der Filmwissenschaft Konjunktur und geben Anlass zu Diskussion. ${ }^{1}$ Elsaesser selbst hat mit zahlreichen Studien zum Frühen Kino seit Anfang der 1990er Jahre nicht unwesentlich dazu beigetragen. Ziel der Medienarchäologie in der Nachfolge Foucaults ist es, das Aufkommen von Medien aus dem vielstimmigen Ensemble ihrer epistemologischen Bedingungen heraus zu erklären und nicht einer historischen Erzählung unterzuordnen. Das Kino, das, wie Elsaesser einmal formuliert hat, technologisch und seinem Realitätsverständnis nach tief im 19. Jahrhundert verwurzelt ist, stellt dafür einen ergiebigen Untersuchungsgegen-

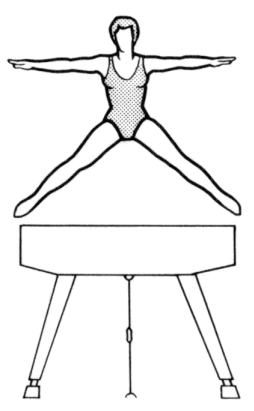

stand dar. Die Einsicht, dass die Epistemologie des Films auf der wissenschaftlichen Untersuchung automatisierter Bewegung, der Entdeckung der fotografischen Reproduktion und der Geschichte von Projektionstechniken beruht, ist von medienarchäologischer Seite kaum je angetastet worden. ${ }^{2}$ Vielmehr wurde diese Grundannahme sukzessive um Forschungen zur Unterhaltungskultur des 19. Jahrhunderts, zum Rezeptionsverhalten eines Massenpublikums und zu Dispositiven der Wahrnehmung ergänzt. ${ }^{3}$

An dieser Ausgangslage ändern auch die beiden aktuellen Film Culture in Transition-Bände nichts. Pasi Väliaho rekonstruiert in seiner Studie Mapping the Moving Image. Gesture, Thought and Cinema 1900 die Epistemologie automatisierter Bewegung im Zeitraum zwischen 1870 und 1920, um zu zeigen, dass das Kino als Formation von Rationalitäten, Körpern und Maschinen zum Motor der Moderne wird. Seine Rekonstruktion orientiert sich an Deleuze' Konzept des Bewegungs-Bildes, das er als Realität verändernde und Geschichte bildende Kraft diesseits und jenseits des Sichtbaren versteht und in Apparaten, künstlerischen Formen und wissenschaftlichen Konzepten gleichermaßen verkörpert sieht. Auch das Verfahren des Mappings und die Darstellungsform des Diagramms fußen auf den Überlegungen von Deleuze und Guattari zur Erschließung von Wissensformationen. In deren Sinn versteht Väliaho das Aufkommen des bewegten Bildes als vielstimmiges Ereignis, das Wissenschaft und Philosophie, Technologie und Ästhetik zusammenführt.

Seine Grundthese einer «fundamental reversability between the media technology and the body" (S. 58) greift auf Walter Benjamin, aber auch auf die von Ernst Kapp in seiner Philosophie der Technik von 1877 entwickelte Auffassung 


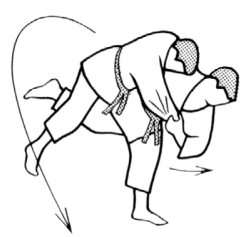

zurück, Medien seien Erweiterungen menschlicher Organe. Väliahos Untersuchung schließt immerwiederwissenschaftliche Konzepte und Experimente (der Physiologie, der Wahrnehmungspsychologie, der Psychiatrie) mit filmischer Ästhetik des Frühen Kinos (Schock, Überraschung, Inkommensurabilität) kurz, um zu zeigen, dass im und durch das Kino die Prozeduren der Macht längst das pure Leben selbst erreicht haben. Dabei geht es Väliaho immer wieder um die grundsätzliche Frage, welche Verbindungen technische Medien mit den menschlichen Sinnen haben und auf welches Körperkonzept sie zurückzuführen sind. Neuropathologische Experimente finden für ihn ihre Fortsetzung im Kino. Seine Studie will nachweisen, dass das technisch produzierte Bewegungsbild die Trennung zwischen dem Bild als psychischer Realität und Bewegung als physischer Realität auflöst. So führe zum Beispiel die kaleidoskopische Visualität der phantom rides des Frühen Kinos zu instabilen Verbindungen zwischen Wahrnehmung und Affekt.

Väliaho entwickelt seine medienarchäologische Untersuchung entlang zentraler Begriffe wie Geste, Rhythmus, Umwelt und Zeit als diskursive Gravitationszentren des Untersuchungszeitraums. Rhythmus etwa wird einerseits als Kraft definiert, die den Zuschauer an die Leinwand fesselt, andererseits als Schlüsselkonzept der rationalisierten Moderne betrachtet und stellt Väliaho zufolge somit ein Modell sowohl der affektiven Ansteckung als auch der sozialen Kontrolle dar. Mit dem Kino entstehen für ihn Kreisläufe von Affizierung und Aktion und daraus wiederum neue Konfigurationen von Zeitlichkeit, die das Denken verändern. Er rekonstruiert dabei vorrangig, wie Vorstellungen des bewegten Bildes das Denken von Friedrich Nietzsche über Henri Bergson und Sigmund Freud bis zu Martin Heidegger selbst dann beeinflusst und geprägt haben, wenn sie es nicht explizit thematisiert haben. Auch zahlreiche Naturwissenschaftler - von Hermann von Helmholtz über Jakob von Uexküll bis zu Albert Einstein - kommen zu Wort. Dabei geht es Väliaho um den Nachweis, dass bereits vor 1900 kein Konzept von Zeit oder Erinnerung ohne eine Vorstellung des bewegten Bildes auskommt. Im Film, der Zeit als Bewegung rhythmisch organisiert, kommt dieses Denken für ihn schließlich zur Positivität.

Das Kino von Georges Méliès dient Väliaho dabei als Beleg für einen entscheidenden epistemologischen Umbruch, der im gewandelten Verhältnis zwischen dem
Belebten und dem Unbelebten, dem Erwarteten und dem Zufälligen, dem Erscheinen und dem Verschwinden zu Tage tritt. Die Moderne ist für ihn durch Plötzlichkeit, Zufall, Unbewusstes und vor allem durch die Unmöglichkeit gekennzeichnet, zwischen Subjekt und Objekt, Realität und Technologie zu unterscheiden. Méliès' magische Transformationen und unerwartete Metamorphosen, in denen sich die Technologie des Kinos selbst offenbare, wertet er als Emblem dieses Umbruchs. Das Ausstellen der kinematografischen Technik gehört jedoch, das hat etwa Tom Gunning immer wieder betont, zum festen Repertoire des Attraktionskinos und ist keineswegs eine Eigenheit der Filme von Méliès.

Für Väliaho sind es aber gerade die Filme von Méliès, die den automatischen Charakter des Bewegungsbildes, seine Fähigkeit zur permanenten De- und Refiguration ausstellen. Auf sie stützt er seine These einer Reversibilität von physischer und maschineller Automation. Auch Körper werden hier zu Automaten und sekundieren die technologische Automation der Bewegung. Nicht von ungefähr hegt Väliaho eine Vorliebe für unwillkürliche Körperbewegungen und nervöse Gesten - Kino und Psychopathologie interessieren sich um 1900 gleichermaßen für motorische Automatismen und ihre Defekte. Dabei erschöpft sich die Rolle des bewegten Bildes für ihn nicht in der Repräsentation von Gesten, deren technologische Positivität ist vielmehr in der permanenten Modulation des Körpers verankert. Väliaho schlussfolgert deshalb mit Bezug auf Giorgio Agambens «Noten zur Geste», dass das Kino die Geste und den Körper in ihrer unbewussten Medialität spiegelt. Das Kino wird in dieser Analogie selbst zu einer Art Geste, die zugleich die Krise der Wahrnehmung und der Repräsentation des Körpers in der Moderne offenbart. Darüber hinaus wird für Väliaho der filmische Raum bei Méliès durch das Zusammenwirken verschiedener Filmtricks zu einer «matrix of conflicting processes» (S. 36) transformiert, einer Matrix, die eine mehrdimensionale Form der Visualität erzeugt. Diese Visualität hebt die räumliche Orientierung des Zuschauers, die Unterscheidung von Größenverhältnissen, Abständen und Platzierungen im Raum auf. Aus der Analyse des filmischen Raums bei Méliès schlussfolgert Väliaho, dass das filmische Bewegungs-Bild zu einer Simulation des Körpers des Betrachters und umgekehrt wird. Unter Simulation versteht er «a new kind of ontological and technological formation" (S.38). Automatisiertes Bewegungs-Bild und Körperautomatismen sind für ihn restlos ineinander überführbar und etablieren veränderte 
Konfigurationen zwischen Wissen und Visualität in der Moderne, in deren Zentrum der Film steht.

Auf Grundlage dieser Argumentation stellt Väliaho immer wieder Verbindungen zwischen kinematografischer Automation und physischen (Reflexe, Rhythmus) bzw. psychischen Automatismen (unwillkürliche Gesten, Tourette-Syndrom) her. Diese an Beispielen des Frühen Kinos erläuterten körperlichen Automatismen wertet er im Anschluss an Anson Rabinbachs Studie The Human Motor als Effekte der Ende des 19. Jahrhunderts entstandenen Bewegungstechnologien. Die Lektüre einzelner Filme dient ihm einerseits als Ausgangsbasis für seine Rekonstruktion von Diskurszusammenhängen, andererseits fungieren genau diese Filme wiederum als Beleg für die rekonstruierte Wissensformation. Dies zeigt sich etwa im vierten Kapitel des Buches, "Paradox Image: Mapping the Unconscious", wo die weiße Leinwand im Film Le mystère des roches de Kador (Frankreich 1912) nicht nur das Trauma seiner Heldin offenbart, sondern der Film das ihr unzugängliche Unbewusste sichtbar macht. Weil Väliahos Beispiele vor allem selbstreflexive Metafilme sind, benutzt er sie zugleich als historische Quelle und Theoriebaustein. Das Aufkommen des Kinos lässt sich so als Teil einer generellen Mechanisierung begreifen, durch die sich das technisch produzierte Bewegungs-Bild und der Körper des Betrachters in einer Zone der Ununterscheidbarkeit befinden. Deshalb widmet Väliaho auch Mareys selbstaufschreibenden Verfahren der Bewegungsanalyse viel Aufmerksamkeit als Beispiel für «non-human observers» (S.40). Diese Ausführungen zeigen, wie viel seine Untersuchung der Medientheorie Friedrich Kittlers verdankt: Dass experimentelle Systeme die Daten erzeugen, die sie angeblich nur aufzeichnen, ist eine Erkenntnis, die nicht zuletzt dessen Schriften verbreitet haben. Auch Väliaho interessiert sich besonders für Aufzeichnungstechniken wie Röntgen- oder Chronofotografie, die ihre eigene Medialität ausstellen und epistemische Dinge hervorbringen. Filmwahrnehmung exerziert in dieser Perspektive nur, was Experimentalanordnungen bereits implementiert und damit vorweggenommen haben. Auch das automatisierte Bewegungs-Bild erscheint so als Effekt seiner automatisierten Aufzeichnung. Seine Epistemologie gehört zum Prozess der allgemeinen Experimentierung des Lebens: Der Körper wird zur dynamischen Oberfläche, auf die verschiedene Kräfte wirken, die gemessen werden können, und das Kino wird zur Technologie dieser Experimentalisierung. Auch Méliès' Filme werden von Väliaho im wahrsten Sinne des Wortes entzaubert: Sie gelten ihm als «diagrammatic vision" (S. 50) des vermessenen Körpers der modernen Naturwissenschaften.

Die Stärke seiner Studie liegt weniger in der Analyse unbekannter Filme oder bisher nicht erschlossener Quellen aus dem Untersuchungszeitraum als vielmehr in der Moderation eines komplexen

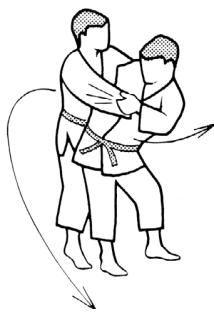
Theoriedesigns, ohne dem gewählten Begriffsrahmen jedoch wirklich neue Facetten abgewinnen zu können. Zudem werden die Auswahl der Quellen und Beispiele sowie die Sprünge zwischen verschiedenen Diskursebenen, die sich durch das transversale Mapping der verschiedenen kulturellen Territorien ergeben, methodisch nicht eigens reflektiert. Die Studie bezieht vor allem die namhaft vertretene deutsche Philosophie sowie die französische und deutsche Naturwissenschaft des 19. Jahrhunderts ein und springt zwischen historischen und aktuellen naturwissenschaftlichen Modellen. Auf diese Weise erschließt sie keine neuen diskursiven Felder, sondern fasst eher den Status quo der medienarchäologischen Forschung der letzten Jahre zusammen und zeigt implizit die Schwierigkeiten einer transversalen Navigation zwischen verschiedenen Diskursfeldern auf.

Kritikwürdig ist schließlich auch Väliahos These einer strikten Reversibilität von Körper und Maschine, die normative Prozesse der Modernisierung in den Vordergrund stellt. Seine Studie geht von Körperkonzepten aus, die vor allem in wissenschaftlichen Laboren und in klinischen Studien entwickelt wurden. Diese biopolitische Vorstellung des modernen Subjekts lässt sich aber nicht ohne Weiteres auf das Sehen von Filmen übertragen. Mit dem Filmpublikum als Masse, der Kinovorstellung als Unterhaltungsform und nicht zuletzt der möglichen Widerständigkeit einzelner Subjekte fehlen der Studie entscheidende Faktoren der Kinokultur, die das Kino erst zum Motor der Moderne machen. Dass gerade die Analyse der Epistemologie des frühen Kinos auf Wahrnehmungsmilieu und Vorführungskontext nur schwer verzichten kann, haben Tom Gunning, Miriam Hansen und andere oft betont.

In dieser Hinsicht nimmt der von François Albera und Maria Tortajada, Filmwissenschaftler/in in Lausanne, herausgegebene Band Cinema Beyond Film. Media Epistemology in the Modern Era, der ebenfalls das interdisziplinäre Netzwerk der Episteme um 1900 untersucht, einen Perspektivwechsel vor. Ausgehend von Foucaults Dispositivbegriff interessieren sich die Beiträge des Sammelbandes 
besonders für Positionen von ZuschauerInnen und HörerInnen bei spektakulären Medienereignissen und rekonstruieren historisch spezifische audiovisuelle Konfigurationen. Wie Albera und Tortajada in ihrer Einführung untersteichen, impliziert dies eine Neuausrichtung des Verhältnisses zwischen Zuschauer (spectator) und Schauspiel (spectacle), genauer: ein neues Verhältnis zwischen Objekt, Apparat, Repräsentation und Zuschauer. Aus diesem Grund üben die Herausgeber auch Kritik an Baudrys und Oudarts Apparatus-Theorien sowie an Kittlers ebenfalls von Lacan informierter Medientrias Grammophon Film Typewriter, aber auch an Crarys Verengung des Dispositivbegriffs auf die Produktion eines normativen Subjekts. Stattdessen entwickeln sie ein Schema der Episteme um 1900, das verschiedene Dispositive zu einem Netzwerk von Beziehungen formt. Dieses Schema wird aus den vielfältigen Beziehungen zwischen Zuschauer, Apparat und Repräsentation ermittelt. Mit dieser Ausrichtung rücken zugleich Fragen in den Vordergrund, die von der Medienarchäologie bisher wenig erschlossene Bereiche der kulturellen Praxis erschließen sollen: Ist der Zuschauer allein oder in einer Gruppe? Ist er bewegt oder unbewegt? Befindet er sich gegenüber einer ihn überragenden Maschinerie oder handhabt er sie? Wird er mit Wirkungen konfrontiert, ohne deren Ursache zu kennen, oder kann er deren Mechanismus durchschauen? Und schließlich: Welchen Zugang hat der Zuschauer zu dem, was er sieht und oder hört?

Damit ist ein weites Feld für medienarchäologische Studien abgesteckt. Diese Erweiterung des bislang in der Filmwissenschaft gebrauchten Dispositivbegriffs soll zu einem Verständnis davon beitragen, was Zuschauer und Hörer in konkreten Medienerlebnissen erfahren. Das erscheint Albera und Tortajada umso wichtiger, als moderne Subjekte in Beziehung zu dem, was sie sehen und hören, verstärkt Erfahrungen der Dezentrierung und der Desorientierung machen. Das Axiom der Mobilisierung als Essenz der Moderne hängt demzufolge weniger von einer allgemeinen physiologischen Disposition als vom Gebrauch konkreter optischer Geräte ab: Wer sich seit dem Anfang des 19. Jahrhunderts eines Zootrops, Phenakistoskops, Thaumtrops oder Kaleidoskops bedient hat, ist Albera und Tortajada zufolge zu einem Wechsel des Sehmodus gezwungen gewesen. In dieser Konkretisierung des Dispositivbegriffs und der Seherfahrungen sehen die HerausgeberInnen den Gewinn ihres Schemas.

Gleichzeitig begreifen Albera und Tortajada wie auch Väliaho das Kino als Fluchtpunkt der sich entfal- tenden Moderne, stellen ihre Rekonstruktion aber auf eine breitere analytische Basis, indem sie verschiedene Diskursebenen miteinander verbinden: Technische Beschreibungen, Schriften von Erfindern, Ingenieuren und Vermarktern sollen ebenso wie Zeugnisse von Zuschauern und Veranstaltern sowie literarische Texte und andere Formen der Fiktion berücksichtigt werden. Mit Väliaho teilen Albera und Tortajada auch das Interesse an Mareys chronofotografischen Studien, dem sie je einen Aufsatz widmen. Das Verhältnis von Augenblick, Belichtung und Intervall steht im Beitrag von François Albera "The Case for an Epistemolography of Montage. The Marey Moment» im Vordergrund. Er wendet sich in seiner Argumentation dezidiert gegen die auf Descartes zurückgehende These einer Analogie zwischen Mensch und Maschine. Für ihn hat Marey weder die Maschine im Körper wiederentdeckt noch den Körper der Maschine angeglichen - im Gegenteil: Die maschinellen Aufzeichnungen mussten sich den Spuren der beobachteten körperlichen Vorgänge anpassen. Der Begriff Mechanismus bekomme daher bei Marey eine eigene Prägung. Die Chronofotografie spiele für ihn auch deshalb eine so große Rolle, weil sie den realen Diskontinuitäten, Zuckungen und Intervallen physischer Bewegungen ähnlich sei. Deshalb habe Marey versucht, die Abfolge der fotografischen Aufnahmen und die Bewegungsphasen miteinander in Einklang zu bringen. Inwiefern Mareys chronofotografische Bewegungsstudien im Sinne Bergsons zu einem anderen Typ von Zeitlichkeit und Subjektivität der Wahrnehmung führen, diskutiert auch Maria Tortajadas Beitrag «The «Cinematographic Snapshot). Rereading Etienne-Jules Marey». Darin zeichnet sie minutiös seine verschiedenen Auffassungen über Moment und Intervall nach. Ihr Aufsatz erhellt, wie Marey versucht hat, die Abstände zwischen den Einzelaufnahmen in einer Serie zu kontrollieren, und rekonstruiert gleichzeitig die epistemologisch wichtige Trennung zwischen fotografischer und chronofotografischer Momentaufnahme: Nicht mehr die Bewegung des fotografierten Objekts sei für letztere die entscheidende Variable, sondern das Intervall der Aufnahmen. Dieser Bruch berührt auch den epistemischen Status des bewegten Bildes. Wie wichtig die Pause zwischen den einzelnen Aufnahmen für Marey wurde, sieht Tortajada auch im Einsatz der fotografischen Flinte belegt.

Solche interessanten Fallstudien lösen aber nur bedingt das Versprechen des Bandes ein, das dispositive Band zwischen Zuschauer, Apparatur und Repräsentation 
neu zu knüpfen. Auch dass die Autorlnnen des Bandes mit jeweils zwei Aufsätzen vertreten sind, erweitert nicht die Perspektiven auf das methodologisch vielversprechend bestellte Diskursfeld. Am überzeugendsten stellt Olivier Lugon den dispositiven Zusammenhang zwischen Spektakel und Zuschauer dar. Er demonstriert in seinem Beitrag «Dynamic Path of Thought» die Mobilisierung des Betrachters durch Ausstellungsdesigns, die Besucher durch den Parcours einer Ausstellung leiten und sie durch wechselnde Ansichten und Perspektiven zur aktiven Teilnahme animieren. Im Zentrum seiner Untersuchung steht neben Bauhauslehrern wie László Moholy-Nagy und Künstlern wie El Lissitzky der Fotograf und Ausstellungsdesigner Herbert Bayer, der nach seiner Emigration aus Deutschland in den USA zahlreiche Ausstellungen konzipiert hat.

Besonders didaktische Ausstellungen haben Lugon zufolge seit den 1920er Jahren mit mobilen Ausstellungsdesigns experimentiert, um Besuchern Inhalte zu vermitteln. Die Überforderung ihrer Sinne wurde dabei oft einkalkuliert. Ausstellungen wurden etwa durch großformatige Fotografien und ganze Wände füllende Fotofriese gezielt als Spektakel inszeniert. Kaum verwunderlich ist, dass in Ausstellungen bald auch Filme vorgeführt wurden. So baute der Architekt Egon Eiermann 1937 für die Ausstellung Gebt mir vier Jahre Zeit, in der Adolf Hitler zum Helden verklärt wurde, ein Kino für 2000 Besucher.

An der propagandistischen Ausrichtung solcher Themenausstellungen zeigt Lugon zugleich das Dilemma moderner Ausstellungsdesigns auf. Je präziser der Weg geplant war, den Besucher durch eine Ausstellung nehmen konnten, umso weniger konnten sie selbst entscheiden, umso mehr wurde ihre Beweglichkeit eingeschränkt. Dies trifft auch auf Norman Bel Geddes New Yorker Futurama von 1939 zu, bei dem die Besucher auf ihren Sitzplätzen wie bei einer Zugreise durch eine futuristische Landschaft bewegt und auf diese Weise wieder zum passiven Staunen verdammt wurden. Lugon versteht es, die dispositive Widersprüchlichkeit von Ausstellungskonzepten, die Besuchern Bewegungsfreiheit versprachen, aufgrund ihrer didaktischen Ausrichtung aber zu ihrer Disziplinierung bzw. Indoktrinierung beigetragen haben, aus der Analyse des Ausstellungsdesigns und historischen Quellen gleichermaßen zu entwickeln.

Alain Boillat unternimmt in seinen zwei Beiträgen den Versuch, den Dispositivbegriff um die Dimension des Akustischen zu erweitern. Er beschäftigt sich unter anderem mit Positionen und Funktionen des Vorlesers bzw. Filmerklärers im Frühen Kino, der eine Mittlerrolle zwischen Publikum und Apparatur einnimmt. Boillat erstellt eine Typologie, um das äußerst heterogene Feld verschiedener Praktiken auf diesem Gebiet zu erschließen. Er unterscheidet dabei zum Beispiel, wo die Quelle der Stimme lokalisiert ist, ob sie sichtbar oder unsichtbar, präsent ist oder aufgezeichnet wurde und berücksichtigt auch, wer die Abspielgeräte in Gang setzt. In einem zweiten Schritt bezieht er Ton- und Bildebene wieder aufeinander und stellt Variabeln ihrer Wechselwirkung vor. Für Boillat gehören die vielfältigen Praktiken der Vermittlung des Films durch das gesprochene Wort, all die sichtbaren oder verborgenen Mittler und aufgezeichneten Stimmen zum Dispositiv des Kinos, des «spoken «cinema»" (S. 227).

Solche Typologien, die zugleich Topologien sind, zeigen die Möglichkeiten auf, die durch die Erweiterung des Foucault'schen Dispositivbegriffs und die Kritik an der Apparatustheorie seit Ende der $1970 e r$ Jahre entstanden sind. ${ }^{4}$ Es gilt dabei in größerem Maße als bisher, die Erschließung von Quellen mit konkreten Fallstudien zu verbinden, um das diskursive Netzwerk zwischen Produzenten und Rezipienten, zwischen Erfindern, Filmgesellschaften, Kinobetreibern, Vorführern, Moderatoren und Zuschauern weiter zu erhellen.

$1 \mathrm{Vgl}$. etwa die umfangreichen Studien von Charles Musser zum Frühen Kino.

2 Immerhin datieren die Materialsammlungen von C. W. Ceram und Friedrich von Zglinicki bereits aus den $1950 e r$ bzw. 196oer Jahren.

3 Zu nennen sind hier z. B. Arbeiten von Vanessa Schwartz, Ben Singer, Martha Braun, Jonathan Crary, Mary Ann Doane.

4 Miriam Hansen, die mit From Babel to Babylon selbst eine viel beachtete Studie zum Publikum des Frühen Kinos vorgelegt hat, hat frühzeitig auf die Rolle der feministischen Filmtheorie und die veränderten Bedingungen des Filmsehens durch Fernsehen und Blockbuster-Kino hingewiesen; vgl. Miriam Hansen, Early Cinema, Late Cinema: Transformations in the Public Sphere, in: Linda Williams (Hg.), Positions: Ways of Seeing Film, New Brunswick, NJ (Rutgers Univ. Press) 1995, 134-152.
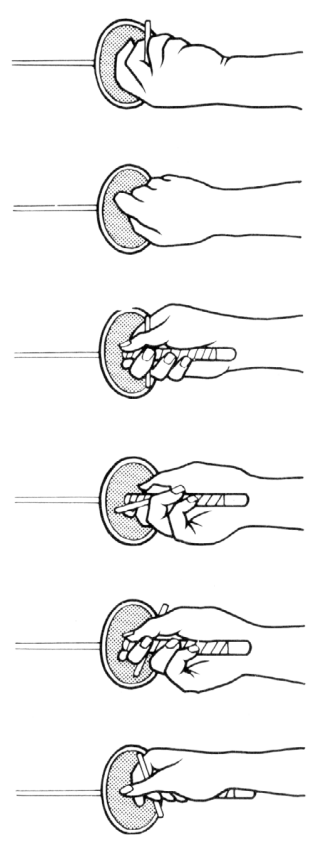